\title{
Optically Controlled Alignment of Nematic Liquid Crystals
}

\section{on Photo-sensitive Polymers}

\author{
Hideyuki Endo, Yukihiro Miyama, Takayasu Nihira, Hiroyoshi Fukuro, \\ Eiichi Akiyama* and Yu Nagase*
}

Nissan Chemical Industries, Ltd., 722-1 Tsuboi-cho, Funabashi-shi, Chiba 274-8507, Japan

*Sagami Chemical Research Center, 4-4-1 Nishi-Ohnuma, Sagamihara, Kanagawa 299-0012, Japan

\begin{abstract}
Alignment properties of nematic liquid crystals (LC) on photo-exposed polymer films have been investigated. Polyimide films prepared from cyclobutane tetracarboxylic dianhydride (CBDA) induced an excellent uniform LC alignment by exposure of linearly polarized UV light. The sensitivity of the photo-alignment of CBDA polyimide films was higher than that of aromatic polyimide films. On the other hand, the LC alignment on fully aromatic polyamide films exhibited the excellent uniformity, although these polyamides contained no photo-reactive groups such as cinnamoyl group. The obtained LC alignment was thermally and optically stable.
\end{abstract}

Keywords: Photo-alignment, Polyimide, CBDA, Polyamide, Sensitivity, Stability

\section{Introduction}

Uniform alignment of liquid crystals (LC) is indispensable for fabricating most liquid crystal displays (LCDs). In order to achieve the uniform alignment, mechanical rubbed polyimide layers have been commonly utilized for commercial LCD production. But this method has several problems such as the generation of dust particles and electrostatic charges, which lower the production yield or the quality of
LCDs. Furthermore, it is difficult to obtain the uniform alignment of LC in vertical alignment LCDs or high resolution LCDs. Therefore, non-rubbing methods are desired to resolve these problems.

Recently, it has been reported that polymer films exposed to linearly polarized UV light are capable of aligning LC without the rubbing process. [1 5] In this technique, the three kinds of polymer systems have been mainly proposed, which are based on 1) 
the photo-isomerization of azo-containing chromophore dispersed in polymer matrix, 2) the photo-dimerization of cinnamonyl or coumarin unit bonded to the polymer as the side group, and 3) the photo-degradation of polyimide films. This LC alignment method using lineally polarized UV light is one of the most favorable candidates to realize the non-rubbing process. However, the sensitivity of these materials by irradiation with UV light and the thermal or optical stability of the LC alignment in LC cells are not sufficient for the practical application.

In this paper, we report the alignment properties on several polymer films by exposure of polarized UV light. And also, the sensitivity of these polymer films by the UV exposure and the thermal or optical stability of the LC alignment on these polymer films are described.

\section{Experimental}

The chemical structures of polymers used in this experiment are shown in Fig. 1 and 2 . The polymer films were prepared on quartz, $\mathrm{CaF}_{2}$, and glass substrates by curing at $180{ }^{\circ} \mathrm{C}$ or $250{ }^{\circ} \mathrm{C}$ for 1 hour. The thickness of films was about $100 \mathrm{~nm}$. The polymer films were exposed to linearly polarized UV light through a band-pass filter $(254 \mathrm{~nm}$ or $313 \mathrm{~nm}$ ) from the normal direction to the substrate in air or vacuum.<smiles>Cc1ccc(Oc2ccc(C(C)(C)c3ccc(Oc4ccc(N5C(=O)C6C(C5=O)C5C(=O)N(C(C)(C)C)C(=O)C65)cc4)cc3)cc2)cc1</smiles>

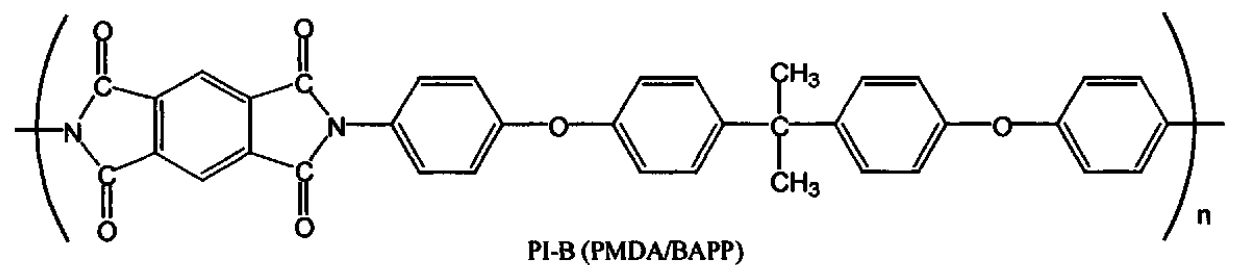<smiles>Cc1ccc(Oc2ccc(C(C)(C)c3ccc(Oc4ccc(N5C(=O)c6ccc(C(=O)c7ccc8c(c7)C(=O)N(C)C8=O)cc6C5=O)cc4)cc3)cc2)cc1</smiles>

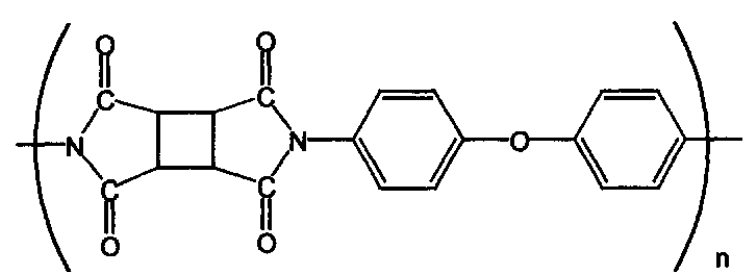

PI-D (CBDA/DDE)

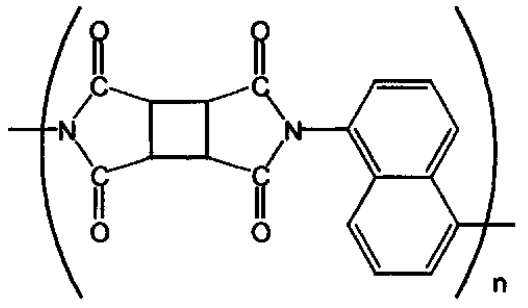

PI-E (CBDA/15DN)

Fig. 1. Chemical structures and abbreviations of polymers 


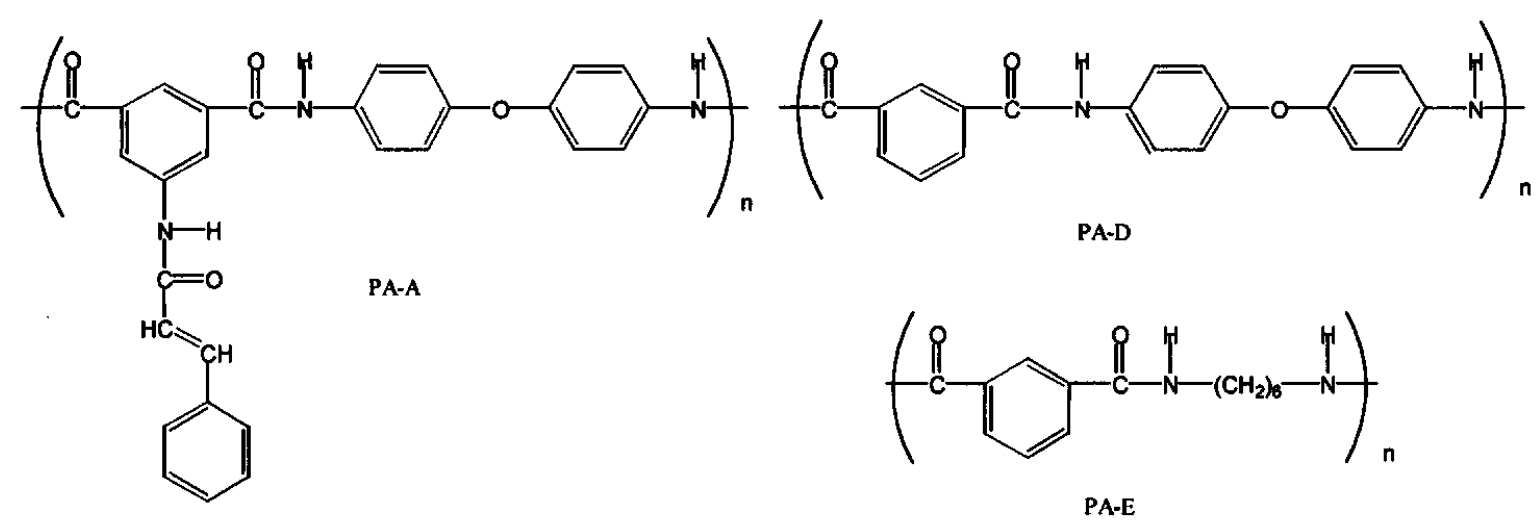

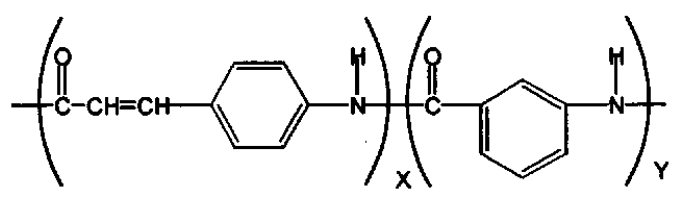

$\mathrm{PA}-\mathrm{B}(\mathrm{X} / \mathrm{Y}=50 / 50)$

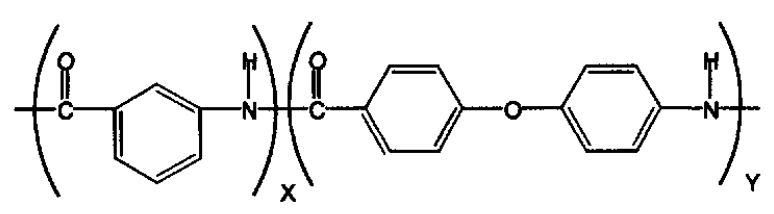

$\mathrm{PA}-\mathrm{C}(\mathrm{X} / \mathrm{Y}=50 / 50)$

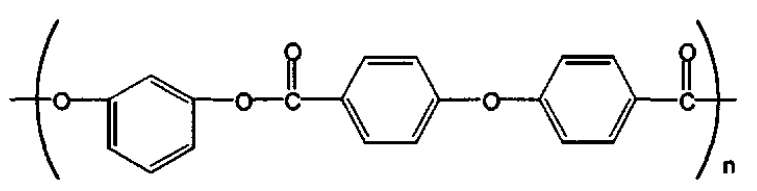

PE

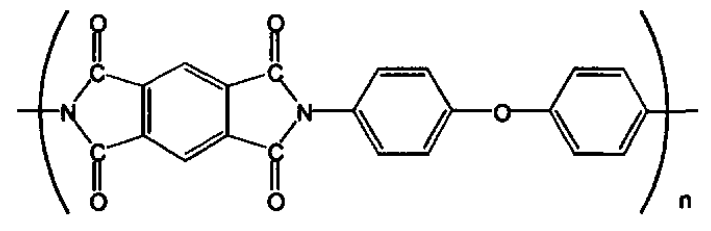

Pl-F (PMDADDE)

Fig. 2. Chemical structures and abbreviations of polymers

The UV light source was a $700 \mathrm{~W}$ super-high pressure mercury lamp, and the UV light was linearly polarized with a UV polarizer made of a multi-coated dielectric film. The irradiation intensity was measured with an ORC UV light measure model MO-02 (detector UV-25 or UV-31).

LC cells were fabricated by assembling two substrates whose directions of electric vectors of incident light were parallel each other, and then nematic LC (ZLI-2293, Merck) were injected into the cells in an isotropic phase. The uniformity of LC alignment was estimated in terms of microscopic observations. The alignment quality of LC was classified into 6 grades, A to $\mathbf{F}$, as follows, $\mathbf{A}$ : uniform and unidirectional alignment without defects, $\mathbf{B}$ : uniform and unidirectional alignment with a little defects, $\mathbf{C}$ : unidirectional alignment with some defects, D: unidirectional alignment with remarkable defects, E: inferior unidirectional alignment with a lot of defects, F: multi-domain alignment.

\section{Results and Discussion}

\subsection{Polyimide [6]}

Table 1 illustrates the grades of LC alignment quality on polyimide films obtained by the UV irradiation of the different irradiation energy. It was found that the alignment uniformity of LC depended on the polyimide structure and the wavelength of exposed UV light. In particular, the dianhydride component of polyimides influenced on the sensitivity for LC 
Table 1. Grade of LC alignment quality on polyimide films.

\begin{tabular}{ccccccccc}
\hline \multirow{2}{*}{ Polyimide } & \multicolumn{3}{c}{$254 \mathrm{~nm}$} & & \multicolumn{3}{c}{$313 \mathrm{~nm}$} \\
\cline { 2 - 5 } \cline { 7 - 8 } \cline { 6 - 8 } & $0.5 \mathrm{~J} / \mathrm{cm}^{2}$ & $1.0 \mathrm{~J} / \mathrm{cm}^{2}$ & $5.0 \mathrm{~J} / \mathrm{cm}^{2}$ & & $1.0 \mathrm{~J} / \mathrm{cm}^{2}$ & $5.0 \mathrm{~J} / \mathrm{cm}^{2}$ & $20 \mathrm{~J} / \mathrm{cm}^{2}$ \\
\hline PI-A (CBDA/BAPP) & B & A & A & & D & C & B \\
PI-B (PMDA/BAPP) & F & E & D & & E & E & C \\
PI-C (BTDA/BAPP) & F & E & D & & E & D & B \\
PI-D (CBDA/DDE) & A & A & A & & D & C & B \\
PI-E (CBDA/15DN) & F & E & D & & B & A & A \\
\hline
\end{tabular}

alignment. PI-A and PI-D consisting of cyclobutane tetracarboxylic dianhydride (CBDA) showed the higher sensitivity at the irradiation of $254 \mathrm{~nm}$, as compared with aromatic polyimides (PI-B and PI-C). On the other hand, the sensitivity of PI-E containing the same CBDA component as PI-A and PID was lower at the irradiation of $254 \mathrm{~nm}$, although PI-E showed the higher sensitivity at $313 \mathrm{~nm}$. This results indicate that the diamine structure of polyimides cause the change of the suitable wavelength for the LC photo-alignment.

To investigate the stability of the LC alignment on PI-D, the LC cells were annealed at $120{ }^{\circ} \mathrm{C}$ for 6 hours or exposed to visible light from a Xe lamp (output 500 W) for 40 hours. In these both cases, the uniform LC alignment was maintained, therefore, the obtained LC alignment was thermally and optically stable.

In order to examine the structural changes of polyimide films after the irradiation of polarized UV light, UV absorption and infrared spectra were measured. As a result, the spectral changes of polyimide films, PI-A, PI-B and PI-E, consisting of CBDA were observed after the
UV irradiation. Therefore, it is considered that the anisotropic photo-degradation would occur in these polyimide films by the irradiation of polarized UV light. Besides, for the aromatic polyimide films (PI-B and PI-C), the spectral changes were hardly observed after the irradiation of $254 \mathrm{~nm}$, and the small spectral changes were observed at $313 \mathrm{~nm}$. These spectral changes were correlated to the alignment properties on polyimide films. The detailed results of photoreactions for polyimide films will be reported elsewhere.

\subsection{Polyamide $[7,8]$}

Table 2 summarizes the grades of LC alignment quality on polyamide films obtained by the irradiation of linearly polarized UV light of $1.0 \mathrm{~J} / \mathrm{cm}^{2}$ energy at $254 \mathrm{~nm}$ and $313 \mathrm{~nm}$, as compared with polyester and polyimide films containing the similar backbone component. PA-A and PA$B$ containing a cinnamoyl group are expected to induce the uniform alignment by the irradiation with linearly polarized UV light. Actually, PA-A and PA-B were capable of aligning LC to some extent, the direction of which was perpendicular to the electric 
Table 2. Grade of LC alignment quality after exposure of light of $1.0 \mathrm{~J} / \mathrm{cm}^{2}$

\begin{tabular}{ccc}
\hline Polymer & $254 \mathrm{~nm}$ & $313 \mathrm{~nm}$ \\
\hline PA-A & D & C \\
PA-B & C & B \\
PA-C & B & A \\
PA-D & C & B \\
PA-E & F & F \\
PE & B & C \\
PI-F (PMDA/DDE) & F & F \\
\hline
\end{tabular}

vector of linearly polarized light. However, unexpectedly, the similar photo-induced LC alignment was also obtained for the films of PA-C and PA-D, although they have no typical photo-reactive group such as cinnamoyl unit. Moreover, the sensitivity for the LC photo-alignment of PA-C was higher than that of PA-A and PA-B, where the uniform alignment of LC was achieved by the irradiation of $0.5 \mathrm{~mJ} / \mathrm{cm}^{2}$ of $313 \mathrm{~nm}$. On the other hand, polyester (PE) and polyimide (PI-F) films, which have similar components to PA-C, induced the LC alignment with some defects and the random alignment, respectively. In addition, PA-E containing an aliphatic group did not give the uniform alignment of LC at all. These results indicated that the alignment property of PA$\mathrm{C}$ and PA-D was the peculiar property for aromatic polyamides.

The stability of LC alignment on PA$\mathrm{C}$ was also evaluated by the same manner as described above. The uniform alignment was held after the heat treatment or the irradiation of visible light to LC cells, therefore, the stability of LC alignment was sufficient for these polyamides.
In general, the photo-oxidative degradation and the photo-Fries rearrangement are well known as the photoreactions of aromatic polyamides. $[9,10]$ Thus, we are considering that both of these reactions took place only in the surface of aromatic polyamides by the irradiation of polarized UV light, because the change of UV absorption spectra of aromatic polyamides before and after irradiation was very small. Furthermore, since the uniformity of LC alignment on PA-C was lowered extremely by irradiation of polarized UV light in vacuum or nitrogen, the photo-oxidative degradation may greatly influenced on the LC alignment. The details of photoreaction for aromatic polyamide films and the mechanism of LC alignment are underway.

\section{Conclusion}

We have investigated the alignment properties of nematic liquid crystals on polymer films exposed to linearly polarized UV light. Polyimide films consisting of CBDA component induced the uniform and stable alignment of LC, and the sensitivity for the photo-alignment was higher than that of aromatic polyimide films. The aromatic polyamide films without typical photoreactive groups also induced the excellent uniformity. Especially, PA-C could align liquid crystals by irradiation of only 0.5 $\mathrm{J} / \mathrm{cm}^{2}$ of linearly polarized $313 \mathrm{~nm}$ light. The induced LC alignment exhibited the thermal and optical stability, which was enough for the practical application. 


\section{References}

1) W. Gibbons, P. Shannon, S. Sun and B. Swetlin, Nature, 351, 49 (1991)

2) M. Schat, K. Schmitt, V. Kozinkov and V. Chigrinnov, Jpn, J. Appl. Phys., 31, 2155 (1992)

3) M. Hasegawa and Y. Taria, $J$. Photopolym. Sci., 8, 703 (1995)

4) J. L. West, X. Wang, Y. Ji and J.R.Kelly, SID 95 Digest, 703 (1995)

5) T. Hashimoto, T. Sugiyama, K. Kato, T. Saito, H. Suzuki, Y. limura and S. Kobayashi, SID 95 Digest, 877 (1995)
6) H. Endo, T. Shinozaki, H. Fukuro, Y. Iimura and S. Kobayashi, $A M-L C D 96$ Digest, 341 (1996)

7) Y. Nagase, E. Akiyama, Y. Miyama, T. Nihira, H. Endo and H. Fukuro, IDW 99 Digest, 21 (1999)

8) Y. Miyama, T. Nihira, H. Endo, H. Fukuro, E. Akiyama and Y. Nagase, IDW 99 Digest, 85 (1999)

9) W. M. Alvino, J. Appl. Polym. Sci., 15, 2123 (1971)

10) D. J. Carlsson, L. H. Gan, and D. M. Wiles, Can. J. Chem., 53, 2337 (1975) 